\title{
Se mouvoir : une question de survie. Témoignage de rencontres avec João Grande et Anna Halprin
}

To Move, It Is a Question of Survival. A Conversation with João Grande and Anna Halprin

\section{Gretchen Schiller}

\section{OpenEdition \\ Journals}

Édition électronique

URL : https://journals.openedition.org/recherchestravaux/745

DOI : 10.4000/recherchestravaux.745

ISSN : 1969-6434

Éditeur

UGA Éditions/Université Grenoble Alpes

Édition imprimée

Date de publication : 28 mai 2015

Pagination : 121-132

ISBN : 978-2-84310-298-1

ISSN : 0151-1874

\section{Référence électronique}

Gretchen Schiller, «Se mouvoir : une question de survie. Témoignage de rencontres avec João Grande et Anna Halprin », Recherches \& Travaux [En ligne], 86 | 2015, mis en ligne le 28 novembre 2016, consulté le 29 octobre 2021. URL : http://journals.openedition.org/recherchestravaux/745 ; DOI :

https://doi.org/10.4000/recherchestravaux.745 
Gretchen SCHILLER

Université Grenoble Alpes

UR LITT\&ARTS

\section{Se mouvoir : une question de survie Témoignage de rencontres avec Joáo Grande et Anna Halprin}

L'artiste américaine Lois Weaver, qui a travaillé avec des seniors en compagnie d'artistes et de chercheuses à l'université de Stanford, dit que certains d'entre eux éprouvent l' "age fright ${ }^{1}$ ", la peur de vieillir, un jeu de mots avec l'expression "stage fright», le trac qui empêche d'agir. Les personnes dont il sera question dans cet article appartiennent à une catégorie différente : il ne s'agit pas d' "age fright» mais d' "age fight» - une combativité, une capacité d'agir à travers tous les âges de la vie.

Ces deux artistes légendaires ont voyagé, l'un (João Grande) d'Itagi au Brésil à Manhattan à New York, l'autre (Anna Halprin) de Winnekta en Illinois à Marin County en Californie. Le premier est un joueur de capoeira qui a quitté son village à dix ans pour suivre son Maître Pastinha de capoeira angola à Salvador de Bahia, la seconde est allée danser à New York et s'est retrouvée à l'université du Wisconsin, le premier département de danse ouvert aux ÉtatsUnis (en 1926) par la biologiste Margaret d'Houbler.

I. Expression prononcée par L. Weaver lors du projet Getting On. A Backstage Tour (2013), mené par L. Weaver, H. Paris et L. Hill avec le AGLOW (Association of Greater London Older Women), au San Francisco Senior Center and Channing House retirement community, Palo Alto. Ce spectacle était produit par le Stanford University Community Engagement Grant et les étudiantes Sukanya Chakrabarti (université Stanford), C. Nolan et L. Mothersole (université Queen Mary, RU). 
João Grande, né João Olivero do Santos² le Is janvier 1933 et Anna Halprin, née Anne Schuman le I3 juillet 1920, partagent des valeurs communes concernant l'importance de la pratique des arts du mouvement. Pour eux, la capoeira et la danse contemporaine ne véhiculent pas seulement des pratiques professionnelles mais surtout une philosophie de la vie. Leurs chemins ne se sont jamais croisés, mais sur cette scène-page, ils se rencontreront.

Deux danseurs.

Deux lutteurs.

Deux survivants.

Pour ma part, je les ai rencontrés tous les deux au cours de mon parcours de danseuse et de chorégraphe : Anna Halprin à l'université de Stanford en juin 20I3, lors d'un atelier qui a eu lieu lors du colloque «Performance Studies International Conference 19 : Now Then, Performance and Temporality" et João Grande dans son studio de Manhattan en 1996 quand je réalisais le documentaire de vidéodanse Camarà ${ }^{3}$. À travers ces rencontres très brèves mais qui m’ont fortement marquée, j'ai été inspirée par la manière dont leurs gestes nous renvoient à notre finitude, tout en nous insérant dans une temporalité cyclique, propre au rituel.

Pendant la journée d'étude "Représenter les âges de la vie dans les arts du spectacle», organisée en 2013 par Ariane Martinez à l'université Stendhal Grenoble 3, je me demandais comment notre compréhension du mouvement s'organise grâce au temps et comment les ressources offertes par le mouvement s'altèrent aussi dans notre corps à cause du temps. La question n'était pas de savoir ce que l'on perd en vieillissant (l'ampleur des mouvements, la tonicité, la mobilité), mais au contraire ce que cela apporte comme ressources kinesthésiques, produits de toute une vie. On pense souvent à la danse en tant que forme artistique des corps jeunes, forts et agiles. Mais ce stéréotype ne limite-t-il pas notre compréhension du rôle du mouvement du corps à tous les âges? Peut-être le vieillissement du corps produit-il dans sa durée une virtuosité kinesthésique à laquelle on prête moins d'attention qu'aux traces visuelles laissées par le temps.

Jouer avec l'âge, pour Anna Halprin et Joâo Grande, en quoi cela consistet-il ? S'agit-il de reconnaittre notre date de péremption et de vouloir mieux vivre en attendant, ou peut-être est-il question de survivre aux épreuves et aux tribulations de la vie? Ou est-ce autre chose encore?

2. Les informations concernant le centre de João Grande sont disponibles sur <http://www. joaogrande.org/mestre.htm> [consulté le oI/03/20I4].

3. G. Schiller, Camarà, documentaire de vidéodanse, produit par le Banff Centre for the Arts, en noir et blanc, 16 min, 1997, Canada, prix Dance on Camera, NY, EU. 
Anna Halprin et João Grande nous aident à éclairer la question du jeu avec l'âge par leur propre vécu professionnel ainsi que par l'importance qu'ils attachent au mouvement comme rituel dans leur vie quotidienne. Pour Joáo Grande, capoeira "é a vida» ("c'est la vie»). D'après lui, on apprend la capoeira en la pratiquant chaque jour ("Com Capoeira eu estou apprendendo cada dia»); c'est ce qu'il a fait, m'explique-t-il, depuis son initiation à la capoeira angola à l'âge de vingt ans jusqu'à ses quatre-vingt-deux ans.

Bien qu'Anna Halprin et João Grande soient issus de contextes politiques et culturels très différents, cet article souhaite mettre en avant leurs affinités. Cette approche comparatiste est contestable car João Grande ne se décrit pas comme un chorégraphe, mais comme capoeirista : la capoeira angola est un art martial, un jeu et une danse. Anna Halprin est danseuse. Mais dans le contexte de cet article, ils forment un duo. Ils continuent à pratiquer leur forme artistique aujourd'hui, âgés respectivement de quatre-vingt-quatorze et quatre-vingt-deux ans, ils partagent des pratiques communes : la porosité entre le corps et l'environnement, la place du rituel et une valeur accordée à la transformation de l'être et de la communauté.

Pour tous deux, les pratiques des arts du mouvement sont des processus de la vie quotidienne. Ils vivent et travaillent au même endroit. Ils visent à construire une communauté, à rester en lien avec le monde naturel et avec le mouvement issu de cette dynamique. Ils valorisent l'expérience de la transformation du corps vécu au détriment des prouesses physiques, quel que soit l'âge qu'on a. Pour eux, la danse ne se limite pas à une maitrise ou bien à un âge donné. D'après João Grande :

On ne devient jamais expert en capoeira : la vie se charge chaque jour de nourrir le terrain de la capoeira. Il n'y a ni début ni fin à la capoeira ${ }^{4}$.

La définition de la danse d'après Anna Halprin présente une certaine similarité avec la philosophie du mouvement de João Grande :

Le mouvement a lieu partout et tout le temps. Il est dans le mouvement de nos cellules, la pulsation de notre sang, le rythme de notre respiration. Il est aussi dans les vagues de l'océan qui monte et qui descend, et dans l'alternance du jour et de la nuit. Le mouvement, c'est la vie, et le mouvement est la source de la danse. Tout corps, qu'il soit jeune ou vieux, et quelle que soit la condition physique, a la capacité de se déplacer, même si c'est seulement votre petit doigt ou un mouvement porté comme une image dans l'œil de votre esprits.

4. J. Grande, entretien avec G. Schiller, Manhattan, EU, I995.

5. "Movement is happening everywhere all the time. It is the motion of our cells, the pulse of our blood, the rhythm of our breath. It is, as well, the ocean waves rising and falling and the alternating 
De plus, Anna Halprin et João Grande se sont donné la mission commune de valoriser la place des traditions et des rituels dans leurs pratiques corporelles. Pour João Grande, la tradition de la capoeira angola n'est pas qu'une histoire du passé mais une situation réelle et un rituel de la vie quotidienne. Quant à Anna Halprin, elle crée des «rituels en collectivité et des cérémonies à partir des situations réelles de la vie ${ }^{6} »$.

Les pratiques d'Anna Halprin et João Grande sont des performances qui recoupent les qualités du rituel définies par Richard Schechner dans le champ de recherche des Performance Studies. Schechner fait un schéma représentant un éventail à l'intérieur duquel chaque branche porte le nom d'une composante de la performance. Ces composantes sont :

les rites et les cérémonies,

le chamanisme,

le surgissement et la résolution de crises,

la performance dans la vie quotidienne, les sports et les divertissements,

les jeux,

les processus de création artistique,

les actes rituels?

Cela implique potentiellement :

Une forme d'efficacité,

le lien avec un Autre absent,

un temps symbolique,

que le Performer soit possédé, en transe,

que le public participe,

que le public adhère et croie à l'événement,

que le jugement critique soit suspendu

qu'il y ait créativité collective ${ }^{8}$.

Le rituel est au cœur de la pratique d'Anna Halprin :

patterns of night and day. Movement is life and movement is the source of dance. Any body, no matter how old or young, in whatever physical condition, has a capacity to move, even if it is just your little finger or a movement carried as an image in your mind's eye." Je traduis. (A. Halprin, Returning to Health, Mendocino, LifeRhythm Books, 2002, p. 23.)

6. "collectively create rituals and ceremonies out of life situations" Je traduis. S. Banes décrit en ces termes le travail de A. Halprin. (S. Banes, Terpischore in Sneakers, Middletown, Wesleyan University Press, 1988, p. 9.)

7. "Rites, ceremonies, shamanism, eruption and resolution of crisis, performance in everyday life, sports entertainments, Play Art-making process, ritualization." Je traduis. (R. Schechner, Performance Theory, New York, Routledge, 1994 [1988], p. xii.)

8. Schéma 4.4 "Link to an absent Other, Symbolic time, Performer possessed, in trance, Audience participates, Audience believes, Criticism discouraged, Collective creativity." Je traduis. (R. Schechner, ibid., p. I20.) 
J'utilise ce mot [rituel] dans son sens très ancien, celui qui signifiait que la danse était totalement associée à la vie d'un groupe humain, et c'est exactement ce que je fais dans mon travail. [...] Lorsque j'utilise ce mot, il signifie toujours pour moi un rapport direct à la vie réelle, ce qui n'est pas mystique du tout mais au contraire très concret... j'utilise aussi le terme de mythologie personnelle, dans le sens où notre histoire personnelle peut devenir une histoire archétypique?

On peut résumer ces propos en affirmant que pour Anna Halprin et João Grande, le rituel est danse : c'est "essentiellement l'achèvement, par l'action, d'une certaine sorte de transformation symbolique de l'expérience ${ }^{\mathrm{IO}}$ ». La pratique du rituel entre en relation avec les cycles de la vie et du monde naturel. Pour Anna Halprin, l'un des exemples les plus parlants de son travail artistique dans la nature correspond à la danse Seasons 2003 où les danseurs sont suspendus dans les arbres de la forêt :

Anna Halprin : Je me souviens qu'étant enfant, lorsque j'étais contrariée, je trouvais ma consolation dans la nature. Il y avait un arbre auquel j'avais donné un nom, j'allais le voir, je lui parlais, je grimpais sur ses branches, c'était comme un ami ${ }^{\mathrm{II}}$.

La relation entre le monde naturel et la compréhension du mouvement est également d'une grande importance pour João Grande :

Gretchen Schiller : João Grande, je vais en France. Est-ce que tu connais un maître de capoeira là-bas?

João Grande : Non, mais tu peux utiliser une plante comme partenaire.

Dans son studio, une forêt de plantes nous transporte dans un paysage brésilien recréant le monde sensoriel du lieu où il a grandi. Lors d'un cours, il me dit :

Savez-vous comment un poisson fonce dans toutes les directions dans l'eau? Pouvez-vous voir les électrons s'agiter dans le réverbère au coin de la rue? Le mouvement est tout autour de nous ${ }^{12}$.

Dans son studio, João Grande m’a montré comment jouer la capoeira (en portugais on dit jogar la capoeira) avec une "plante-partenaire», puis il m'a raconté une anecdote : lorsqu'il n'avait personne avec qui danser dans les champs au Brésil, ses partenaires étaient des plantes. Ce que j’en ai compris,

9. Entretien entre J. Caux et A. Halprin (J. Caux, A. Halprin, À l'origine de la performance, Lyon, Panama musées, 2006, p. I24).

Io. Citation de l'anthropologue D. Williams dans l'article de J. Hanna, «Pour une compréhension des humains à travers l'étude anthropologique de la danse" [parution originale en anglais en 1979], dans A. Grau et G. Wierre-Gore (éd.) Anthropologie de la danse. Genèse et construction d'une discipline, Pantin, Centre national de la Danse, 2006, p. II7.

II. J. Caux, À l'origine de la performance, ouvr. cité, p. I23.

I2. Conversation entre J. Grande et l'auteur à New York en 1996 dans son studio à Manhattan. 
c'est que la capoeira nous habite. Elle prend corps dans notre vie au delà des nécessités matérielles de la pratique. On trouve le moyen de prendre pour partenaire la nature. Autrement dit : on crée le rituel avec les ressources qui sont à notre disposition.

João Grande, qui a quitté les siens à dix ans, a dû apprendre tôt à survivre jour après jour en faisant de petits métiers. Il a trouvé sa vocation en rencontrant Maître Pastinha, né Vicente Joaquim Ferreira Pastinha (I889-I99I), qui a créé en I94I la première école de capoeira angola, le Centro Esportivo de Capoeira Angola, et écrit le premier livre sur le sujet : Capoeira Angola.

João Grande a été le témoin de la déchéance de son Maître Pastinha qui a perdu son studio puis est mort aveugle, dans la pauvreté et dans la rue. Avait-il en tête, en 1970, quand il est allé enseigner aux États-Unis, que son studio de Manhattan pourrait devenir le sanctuaire où survivrait la tradition de la capoeira angola, transmise par son maître?

Pour Anna Halprin, c'est différent. À l'âge de trois ans, elle dansait à la maison, et par la suite, elle a suivi des cours dans la lignée d'Isadora Duncan ${ }^{13}$. Dans le documentaire de Ruedi Gerber Breath Made Visible (2009), on apprend que les premiers souvenirs de danse d'Anna Halprin sont les rituels de prières dansés par son grand-père juif, et qu'ils ont marqué sa carrière. Depuis, elle cherche une danse - un rituel aussi spirituel que celui de son grand-père. Elle a quitté les siens pour étudier la danse avec Margaret D'Houbler, qui était très marquée par Mabel Todd, l'auteure de Thinking Body ${ }^{14}$. D'Houbler a eu une influence essentielle sur les programmes de danse universitaire aux États-Unis et son approche anatomique et fonctionnelle du mouvement est encore très présente dans le travail d'Anna Halprin. En tant que chorégraphe et enseignante $^{\text {is }}$, celle-ci se centre moins sur la spécificité d'une technique comme les chorégraphes Martha Graham et José Limon, que sur la mise en place des conditions favorables à la libre expression corporelle et sur le changement de l'état intérieur de ses danseurs. Anna Halprin a quitté Manhattan en 1955 pour trouver son lieu d'élection : une scène en plein air, que son mari, le paysagiste architecte Lawrence Halprin, a construite pour elle dans leur jardin.

Elle a utilisé comme approche chorégraphique la méthode RSVP (Resource Score Valuaction Performance), développée par son mari pour stimuler la créativité en urbanisme. Avec le RSVP, les chorégraphies peuvent aboutir à une partition très précise ou à une partition très libre. Une grande variété de

I3. L. Worth and H. Poynor, Anna Halprin, Londres, Routledge, 2004, p. 2.

I4. Cet ouvrage, écrit en 1937, a été traduit en 2012 en français sous le titre Le Corps pensant.

I5. Dans son studio en Californie A. Halprin a formé S. Forti, Y. Rainer, T. Brown et R. Morris entre autres. 
chorégraphies est possible : des danses sont créées à partir de tâches précises, d'autres correspondent à des projets communautaires citoyens (comme Seniors Rocking [2005] qui s'est déroulé sur le lagon de Grassy Island avec Anna Halprin et soixante personnes âgées du Redwoods Community of Seniors and the Sunshine Club au Marin County, assises dans des rocking-chairs), d'autres expériences ont lieu avec des patients en soin intensif, comme l'Intensive Care (2006).

Pour Anna Halprin, « [1] a tâche à accomplir n'est rien d'autre qu'une technique qui permet à chacun d'être attentif au mouvement qu'il fait et à sa résonance avec l'environnement dans lequel il se trouve à ce moment très

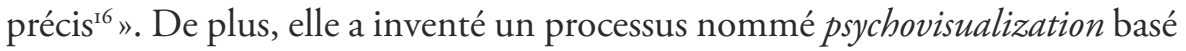
sur le dessin et le mouvement, qu'elle a utilisé elle-même pour guérir d'un cancer. Elle a été influencée par les enseignements de Fritz Perl qui lui ont appris à apprécier les transformations et influences possibles entre le corps et l'esprit.

En ce qui concerne João Grande, c'est la nature et Mestre Pastinha qui lui ont appris la capoeira. Les rencontres avec Mestre Pastinha à Salvador ont changé sa vie. La transmission de la capoeira angola est passée par Mestre Pastinha qui lui-même avait été formé par un esclave, Beneditto, venu d'Angola. La capoeira, art martial, danse, jeu, en théorie, est une évolution de la danse nommée Zebra en Angola, danse pratiquée par les Africains arrivés au XVI ${ }^{\mathrm{e}}$ siècle au Brésil. Elle est composée de chansons, de musique et de mouvements. La musique polyrythmique est jouée par trois birimbaos, un agogo, un atabaque, un pandeiro et un reco-reco, les textes sont chantés par les capoeiristas euxmêmes à partir d'une narration concernant la survie et l'identité du peuple. On peut relever des signes et des symboles dans les rythmes musicaux. Par exemple, on suppose que le rythme musical nommé la cavalaria («la police») était un signal pour alerter les esclaves de l'arrivée de la cavalerie. La cavalaria est une chanson qui imite le pas des chevaux. Dès qu'ils l'entendaient, les esclaves remplaçaient les gestes de lutte par des pas de danse.

Jouer la capoeira implique de faire partie de la roda, un cercle de participants chantants et jouant d'un instrument. Jouer la capoeira revient à se mouvoir de manière circulaire en interaction avec son partenaire, se mouvoir de haut en bas et autour du corps de l'autre en dessinant de nouveaux itinéraires de rencontres des corps. Dans le jeu de roda, il n'y a ni gagnant ni perdant. Le but est simplement de se transformer, d'expérimenter l'aché (notion que je commenterai dans la suite de l'article).

Les mouvements codifiés sont appris : ginga, negativa, rolé, rabo de raiz, troca de pé, cabécada, cadeira, aù, balança, bananeira et la meia lua de frente, mais le jeu de capoeira est improvisé. Plus de verticalité, le corps est constamment

I6. J. Caux, À l'origine de la performance, ouvr. cité, p. I25. 
en spirale, la tête en bas, la tête en haut. Ce ne sont que des arcs, des attaques, des contre-attaques, des lignes droites, des roues et leurs rayons qui traversent le corps des joueurs.

Les vocabulaires gestuels de la capoeira angola et de la danse d'Anna Halprin ne se ressemblent pas. Mais éloignons-nous des traces visuelles et de la danse et déplaçons notre attention vers l'état sensoriel, subjectif et kinesthésique de leurs pratiques. Au cours de l'été 20I3, j'ai participé à un atelier avec Anna Halprin, et j'avais l'impression de voir une femme de soixante ans danser (elle en a quatre-vingt-quatorze). Nous nous sommes échauffés au son du tambour joué par un membre de sa compagnie et par la suite, après un travail collectif, nous avons réalisé la chorégraphie de la Planetary Dance (Danse planétaire). Cette chorégraphie fait circuler trois groupes en cercles concentriques : des marcheurs, des joggers et des sprinters.

La notion de cercle est toujours très présente dans le travail artistique et pédagogique d'Anna Halprin. Son solo Prophetess (1955), basé sur des mouvements circulaires, ressemble à ceux des derviches turcs. Dans ses cours, elle demande aux étudiants de sentir le corps-espace comme une pierre qui frappe l'eau : «il faut sentir les cercles autour de votre $\operatorname{corps}^{17}$ ». La chorégraphe et étudiante d'Anna Halprin, Simone Forti, se souvient que dans ses cours, Anna demandait aux danseurs de se promener dans un cercle et de se concentrer sur les sensations de leur corps ${ }^{18}$.

Du corps aux orbites planétaires, la question du cercle est omniprésente dans son travail. La Planetary dance ${ }^{19}$ a commencé dans les années 1980 chez Anna Halprin à Marin County. La motivation de cette danse vient de l'angoisse générée par l'assassinat de sept femmes sur la montagne Tamalpais à côté de chez elle. Pour exorciser cette peur, Anna Halprin a proposé aux habitants de Marin County de danser en groupe. Cette danse, inspirée par celles des Indiens qui habitent à proximité, avait pour but la prise de conscience et la guérison de Marin County. C'est ainsi que les habitants ont pu retrouver leur montagne, après l'arrestation du tueur, deux ans après leur danse ${ }^{20}$.

17. R. Gerber, Breath Made Visible, Revolution in Dance Anna Halprin, Zurich et New York, ZAS film AG, 2009.

I8. "Sometimes it would be a matter of doing an activity that would set you up or help you induce the dance state. It could be just walking around in a circle and focusing on the sensations in your body." Je traduis. (S. Banes, Terpischore in Sneakers, ouvr. cité, p. 23-24.)

I9. Site du Planetary dance : <http://www.planetarydance.org/ consulté le I ${ }^{\text {er }}$ mars, 20I4>.

20. Entretien entre K. Dychtwald et A. Halprin le 5 octobre 20I2, Big Sur, Californie au Esalen Institute's pour les cinquante ans de l'institut de Esalen, disponible sur <https://www. youtube.com/watch?v=SiUEo-NveZo> [consulté le 02/04/20I4]. 
Aujourd'hui, trente ans après, la Danse planétaire est devenue un rituel international qui a comme objectif de danser pour évoquer un problème grave ou défendre une cause importante. En effet, depuis l'épreuve de son cancer, Anna Halprin a décidé de s'engager par la danse. Pendant l'atelier, nous avions compris que ceci lui tenait à cœur. Chacun a été amené à se prononcer devant le groupe sur sa propre raison de danser.

La capoeira angola, comme la Danse planétaire, est un jeu en cercle. Notre participation à la chanson crée un trait d'union entre l'héritage du passé des esclaves, les anciens maîtres de capoeira mais aussi l'histoire personnelle du joueur. L'individu s'inscrit comme membre d'un groupe, d'une communauté.

En capoeira angola, le mouvement peut être décrit comme un combat, un mime des jeux animaliers et/ou des jeux de camaraderie. De la même façon, pour Anna Halprin, «[1]a danse peut consister, comme le jeu, en des rituels de rébellion, ou des exutoires pour la déviance, une façon de représenter un segment du psychisme ou du monde pour le comprendre ou pour y faire face ${ }^{21}{ }^{\prime}$.

Pour la Danse planétaire et la roda de capoeira angola, ce qui importe n'est pas l'apparence du corps, mais la transformation du corps par le rituel, un phénomène que le philosophe Jose Gil nomme le corps paradoxal:

Un corps humain, parce qu'il peut devenir animal, minéral, plante, se fondre dans l'atmosphère, dans un trou, dans l'océan, peut devenir le mouvement pur. [C'est cela,] le corps paradoxal ${ }^{22}$.

João Grande et Anna Halprin habitent et m'ont fait habiter la notion de corps paradoxal. En réfléchissant plus précisément à la perception kinesthésique qui a été la mienne lorsque j'ai interprété la Danse planétaire (2013) et pris part à la roda de capoeira (1996), je peux dire que ma conscience corporelle a oscillé entre une perception du corps collectif du groupe et celle de mon corps individuel par les techniques polyrhythmiques et circulaires. L'entre-(je)uxespace-rythme était le processus le plus marquant de ces expériences, et il a transformé mon état corporel et l'a déplacé vers un corps paradoxal.

Dans les deux cas, mes souvenirs corporels avec Anna Halprin et João Grande sont marqués par le son du tambour, un instrument qui résonne

2I. "Thus dance may be, like play, rituals of rebellion, or cathartic outlets for deviance, a way of representing a segment of the psyche or world to understand or cope with it. " Je traduis. (J. Hanna, To Dance is Human, a Theory of non Verbal Communication, Chicago, University of Chicago Press, 1987, p. 27.)

22. "A human body because it can become animal, become mineral, plant, become atmosphere, hole, ocean, become pure movement. That is: a paradoxical body. " (J. Gil, «Paradoxal Body», dans A. Lepecki et J. Joy [éd.], Planes of composition. Dance, Theory and the Global, Londres, New York, Calcutta, Seagull, 2009, p. 94.) Le texte de J. Gil, paru en 200I, est traduit du portugais en anglais par A. Lepecki et J. Joy dans cet ouvrage. Je traduis. 
fortement dans le bassin. Les inscriptions physiques de la partition rythmique, polyphonique et polymorphique sont plus nuancées. Mon attitude intérieure (notion tirée de Laban ${ }^{23}$ ) était composée des micromouvements de mon corps et de ceux des autres. Ici, j'ai fait l'expérience du fait qu' “ [à] chaque transformation du régime énergétique, se produit une modification correspondante de l'espace ${ }^{24} »$. On devient également corps multiple et corps paradoxal lorsque «[la] danse se compose d'une succession de micro-événements qui transforment sans cesse le sens du mouvement ${ }^{25}{ }^{\prime}$. Avec ces expériences corporelles, on voit bien comment le rythme du rituel est «un circuit compliqué aux matrices changeantes capable de transformations instantanées et rapides ${ }^{26} »$. Dans les deux cas, on ressent le circuit rythmique du collectif qui modifie notre transformation corporelle et l'attitude intérieure de notre état individuel, nommé en capoeira angola l'Aché:

L'Aché est la force magique qui déplace toutes les choses dans l'univers selon les religions africaines du Brésil. Elle existe dans tous les royaumes de la nature et peut être transmise par des rituels spécifiques. Bien que la capoeira n'ait aucun rapport direct avec la religion, les capoeiristas, comme la majorité des Brésiliens, sont liés d'une façon ou d'une autre à des rituels afro-brésiliens. L'Aché dans la capoeira signifie la connexion avec les racines, c'est une énergie spéciale pouvant être développée par n’importe quel capoeirista. Souhaiter "Aché» à quelqu'un revient à lui souhaiter bonne chance ${ }^{27}$.

Que l'on pratique la capoeira angola traditionnelle ou la Danse planétaire contemporaine, on est dans un processus qui transforme notre état grâce aux rituels circulaires. Ce qu'Anna Halprin et João Grande accomplissent est sans doute une manière de nous mettre en éveil et de nous rappeler que la transformation à travers les âges de notre corps est intemporelle.

23. Rudolph V. Laban (1879-1958) nomme «inner attitudes» les états corporels issus des configurations d'efforts-shape. Les états qu'il énumère sont les suivants : awake (éveillé), dream (rêve), stable (stable), mobile (mobile), near (proximal) et remote (lointain).

24. "To every transformation of the energetic regime there is a corresponding modification of the space of the body. " Je traduis. (J. Gil, "Paradoxal of Body", art. cité, p. 92.)

25. "Dance is composed of a succession of micro-events that ceaselessy transform the movement's meaning." Je traduis. (Ibid.)

26. "a complicated circuitry capable of instant transformations and swift shifts of matrices". Je traduis. (J. Hanna, "Pour une compréhension des humains», art. cité, p. 29.) Cette notion de «matrice» est empruntée à Schechner.

27. "Aché (axé, asé) is the magic force that moves all things in the universe according to the African religions in Brazil. It exists in all realms of nature and can be transmitted through specific rituals. Although Capoeira has no direct connection with religion, the capoeiristas, as the majority of the Brazilians, are related one way or another with Afro-Brazilian rituals. Aché in Capoeira means the connection with the roots, a special energy to be developed by any capoeirista. To wish aché to someone means to wish good luck.» (B. Almeida, Capoeira: A Brazilian Art Form, History, Philosophy and Practice, Berkley, North Atlantic Books, I986, p. 6.) 
Pour eux, l'âgentivité (l'âge et l'agentivité) est une question de survie. La capoeira angola vient de la nécessité de survivre contre l'esclavagisme au Brésil. Pour João Grande, la survie coule profondément dans les veines de tous ceux qui ont apporté la danse du Zèbre, des pays d'Afrique de l'Ouest au Brésil. Quand on joue la capoeira, on éprouve ce combat. Aujourd'hui, João Grande incarne la capoeira angola et transmet la tradition comme un rituel du quotidien.

En ce qui concerne Anna Halprin, son dévouement à la danse avec sa Danse planétaire est lié à sa nécessité de survivre aux épreuves qu'elle a vécues à travers les âges de sa vie. "Avant je vivais pour créer, maintenant je crée pour vivre", m'a-t-elle dit pendant notre atelier. João Grande et Anna Halprin utilisent tous les deux leurs connaissances kinesthésiques pour atteindre l'essence de l'être humain. Pour Anna le Life / Art Process, qu'on pourrait traduire comme le «processus entre la vie et l'art", advient lorsque "l'expérience de vie s'approfondit, l'expression artistique personnelle se développe, et quand l'expression de l'art se développe, les expériences de vie s'approfondissent ${ }^{28}$ ». Pour Joáo Grande, la seule constante est le mouvement, la capoeira est la vie. Il faut continuer à se mouvoir chaque jour, dit-il. Et par cette pratique et cet entraînement, il reste lui-même aussi un apprenti. Avec sa voix confiante et grave, il m'a dit : "eu aindo tou apprendendo" (j’apprends encore) ${ }^{29}$.

Lors d'un échange de mails, j'ai demandé à Anna Halprin si elle pouvait nommer les ressources kinesthésiques liées à son âge. Elle m’a répondu, à quatre-vingt-quatorze ans ${ }^{30}$ :

Désolée de vous décevoir, mais la réalité est que je ressens plus les pertes [liées à l'âge] qu'un quelconque gain. Perte d'équilibre, de la force, de la flexibilité et de la mémoire. Cependant, je reste active par l'enseignement, mes performances et l'écriture... Je nage pour garder de l'endurance. Je fais des mouvements pour maintenir mes forces et la coordination de mes membres. En bref, je continue d'être active physiquement, je continue à apprécier la danse sous toutes ses formes et je me sens chanceuse. Je suis encore ici, peut-être un peu plus sage ${ }^{31}$.

28. "as life experience deepens, personal art expression expands, and as art expression expands, life experiences deepens" Je traduis. (A. Halprin, Returning to Health with Dance, Movement and Imagery, Mendocino, LifeRhythm Books, 2000, p. 20).

29. J. Grande, discussion téléphonique avec G. Schiller, le I3 août 2014.

30. Pour approfondir la question de la danse et l'âge, on pourra se référer au chapitre to de l'ouvrage. J. Ross, "Choreographing Disappearance: Dances of Aging 1992-2006", dans Experience as Dance Anna Halprin, Berkeley, University of California Press, 2007.

3I. "Sorry to disappoint you but the fact is I experience more loses then any gain. Loss of balance, strength, flexibility and memory. However, I stay active teaching, performing and writing. I swim to keep up endurance. Do some strengthening movements and practice coordination. In short, I keep active physically, continue to enjoy dance in its many forms and feel lucky. I am still here, perhaps 
Pour les artistes dont il a été question dans cet article, jouer (avec) l'âge ne consiste pas à représenter un âge, mais à rendre présente leur corporalité par une pratique quotidienne. Pratiquer et créer des rituels kinesthésiques, se mouvoir chaque jour est pour eux une façon de survivre à travers les âges. L'âgentivité se pratique.

a little wiser.» Je traduis. (A. Halprin, correspondance par courriel avec G. Schiller, le to juin 20I4.) 